\title{
Congenital Insensitivity to Pain and Musculoskeletal Involvement: A Case Report and a Mini Review
}

\author{
Nadeem Ali ${ }^{1 *}$, Shabir A Dhar ${ }^{2}$, Kafeel Khan² and Naseer A Mir ${ }^{2}$ \\ ${ }^{1}$ Hospital for Bone and Joint Surgery, India
}

${ }^{2}$ SKIMS Medical College and Hospital, India

*Corresponding author: Nadeem Ali, Hospital for Bone and Joint Surgery, Mughal Mohalla, lalbazar Srinagar, Jammu and Kashmir-190023, India

Submission: 㭗 June 15, 2018; Published: 觜 August 13, 2018

\begin{abstract}
Congenital insensitivity to pain is a rare syndrome characterized by absence of sensations of pain and temperature. These children present with self mutilation of tongue, lips and fingers and musculoskeletal complications in the form of multiple and recurrent fractures, dislocations, auto amputations, ulcers of the extremities, infections in the form of soft tissue abscess, osteomyelitis and septic arthritsis, charcot joint arthopathy, heterotrophic ossification, avascular necrosis and deformities. We here present a six year old boy with congenital insensitivity to pain with anhidrosis who had selfmutilation of lips, tongue and fingers and presented with osteomyelitis of distal humerus with septic arthritis of elbow joint that resulted in destruction of the proximal radius and ulna. We also review the literature for the musculoskeletal complication seen in congenital insensitivity to pain.
\end{abstract}

\section{Introduction}

Pain is an unpleasant sensation but a protective mechanism that limits the tissue damage by a natural withdrawal reflex. Absence of this pain pathway can result in persistent trauma to the tissues by various obnoxious agents like mechanical, chemical, thermal and other similar stimuli [1,2]. Most of the pain insensitivities are acquired like peripheral neuropathy of diabetes mellitus, syringomyelia, and leprosy [1,3]. Congenital cases include congenital indifference to pain and congenital insensitivity to pain (CIP). In CIP the painful stimulus does not reach the central nervous system due to abnormality in the pathway while as in congenital indifference the pain pathway is intact but there is failure to react in a normal defensive manner to a painful stimulus rather it is manifested by change in pulse rate, respiratory rate and blood pressure. Congenital indifference is significantly associated with problems in olefaction [1-4].

Congenital insensitivity to pain with anhidrosis (CIPA) was initially described by Dearborn in 1932 and systematic description of same was given by Swanson in 1963 [5]. It was Dyck [6] who introduced hereditary sensory autonomic neuropathy (HSAN) as an entity and classified it into five types (HSAN-I to HSAN-V) based on the clinical presentation, mode of inheritance, pathology and molecular basis in 1984. CIPA is labelled as HSAN type IV and a characteristic feature is anhidrosis attributed to lack of innervations of sweat glands which is responsible for problems in regulation of body temperature. $20 \%$ of these patients usually die within first three years of life due to repeated attacks of hyperpyrexia [7-9]. CIPA is a rare entity with a reported incidence of 1 in every 125 million new births and an autosomal recessive pattern of inheritance $[3,10]$. Here we present a case of CIPA with septic arthritis of the elbow joint with multiple musculoskeletal abnormalities and also review the literature pertaining to musculoskeletal presentation of this rare syndrome.

\section{Case Report}

A six year old boy was brought by his parents to the accident and emergency department of our hospital with the complaint of fever and painless swelling of the left elbow for the last two weeks for which a consultation of a local doctor was sought who had advised some oral antibiotics and anti-pyretic drugs. There was no history of respiratory tract infection or any local trauma to the elbow. The child was born full term by normal vaginal delivery of a consanguineous marriage and was third in number. The perinatal period was uneventful. There was significant past history of repeated episodes of hyperpyrexia in the past for which the child was hospitalised number of times. Besides there was history of self mutilation with infliction of injuries to fingers, tongue and lips. There was also history of ulcers involving feet and hands which heeled over a long period of time. There was no history of similar type of involvement in any of the siblings or any other family member. On examination the child was febrile with a pulse rate of 100 beats per minute. The child had some behavioural abnormality with mild mental retardation. Examination of the oral cavity revealed loss of all the teeth with healed ulcers over the tip of the tongue (Figure 1a). There were healed ulcers over the helix of the external ear on both the sides with loss of part of the superior part of the helix on the 
right side (Figure 1b). There was amputation of the tips of multiple fingers of both the hands (Figure 1c). There were multiple healed scars visible on both the hands and feet. Both feet were deformed with a rocker bottom type of deformity (Figure $1 \mathrm{~d} \& 1$ e). On local examination, there was a gross swelling of the left elbow which was more prominent on the posterior aspect. The local temperature was raised and the swelling on the posterior aspect was fluctuant. There was no local tenderness and all the movements at the elbow were within normal range and painless. Distal neurovascular status was ok. Detailed neurological examination was not possible because of lack of co-operation but grossly motor functions as well as deep tendon reflexes were normal. Different sensations could not be checked but sensation of pain and temperature was absent as demonstrated by lack of withdrawal on exposure to these stimuli. A radiograph of the left elbow revealed well developed old periosteal reaction on the posterior surface of the distal humerus (Figure 2). Blood investigations had a raised leukocyte count with a predominant neutrophillia. The serum $\mathrm{C}$ - reactive protein levels were positive and raised.
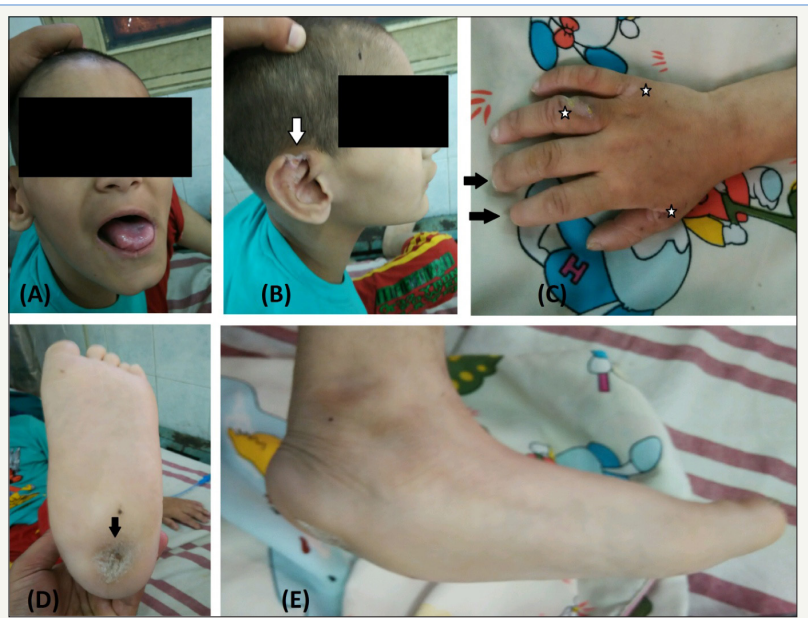

Figure 1:

1a: Healed ulcers of tongue and lip with loss of all the teeth.

1b: Loss of superior part of helix of the external ear (arrow).

1c: Scars on the dorsum of hand (stars) with auto amputation of index and middle finger tips (arrows). 1d: Scar on plantar aspect of heel (arrow).

1e: Rocker bottom deformity of the foot.
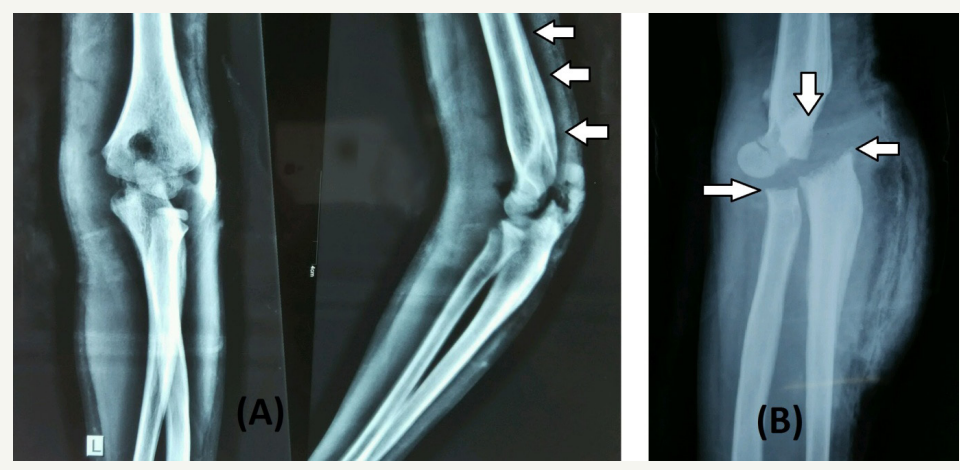

Figure 2:

2a: Radiograph at presentation showing periosteal reaction on posterior aspect of distal humerus (arrows).

2b: Radiograph of elbow at 3 months follow up showing destruction of radial head, olecranon, coronoid and traochlea.

These findings were suggestive of infectious pathology but lack of local signs of tenderness and presence of free movements at the elbow were contradicting each other. Aspiration of the elbow joint was planned and frank pus was aspirated from the posterior aspect. Diagnosis of osteomyelitis of the distal humerus with septic arthritis of the elbow joint was made and was planned for incision and drainage. The Distal humerus and the elbow joint were approached by posterolateral approach and about $100 \mathrm{ml}$ of pus was drained from elbow joint as well as from underneath of the triceps. The posterior surface of the distal humerus appeared rough with visible new bone formation. Copious lavage with normal saline was given and wound was kept partially open for free drainage. The patient was put on parentral antibiotics. The pus sent for culture sensitivity cultured Staphylococcus aureus and bone biopsy that was taken 
from distal humerus had features suggestive of osteomyelitis. The general condition of the patient improved postoperatively but there was persistent discharge from the wound without any signs of healing of the wound at three weeks following which a lavage and debridement was done and a low pressure vacuum assisted closure (VAC) dressings were used for a week after which signs of heeling and appearance of granulation tissue was seen. The wound heeled completely in next six weeks without any need for secondary procedure. At the final follow up range of motion at elbow was well maintained with little instability on stress tests. A radiograph of the elbow revealed complete destruction of the radial head, olecranon, coronoid and trochlea of the distal humerus (Figure 2). Despite the amount of destruction elbow function was relatively preserved because of absent pain sensations which allowed early range of motion at the elbow joint. Nerve conduction studies done at follow up had normal sensory and motor nerve conduction velocities. Sural nerve biopsy was planned but the parents did not give consent for the same.

\section{Discussion}

Congenital insensitivity to pain (CIP) is a very rare entity with most of the literature confined to case reports and short series. Cases have been reported from different parts of the world but most of these cases are from Japan, America and Israeli Bedouin $[11,12]$. Patients with CIPA also known as HSAN type-IV classically have presentation after birth with episodes of hyperpyrexia due to anhidrosis and consequently failure of temperature regulation $[8,13]$. Our patient had similar history with frequent hospital admissions for hyperpyrexia. The problem usually starts with appearance of dentition when the child starts biting lips, tongue and fingers leading to self mutilation [14]. And it is usually the period parents seek medical attention and diagnosis of pain insensitivity syndrome is made. Another milestone when problems start is when the child starts walking and gets exposed to increased mechanical load, making him or her prone to repetitive trauma in the absence of the protective pain pathway [15].

Patients with CIP usually present with musculo-skeletal problems and there is a spectrum of musculo-skeletal involvement. One of the early and important presentations is self mutilation of the body parts which commonly involve tongue, lips and fingers. Mutilation of the fingers results in ulcers of the finger tips and auto amputation. Radiologically one may see destruction of the distal phalanx and is known as osteoacrolysis $[16,17]$. Due to insensitivity decubitus ulcers or pressure sores may develop around elbows, knees, feet and even hands. Burn injuries are also very commonly seen due to lack of temperature sensations [18]. The ulcers may get secondarily infected and pave way to formation of soft tissue abscess and even osteomyelitis in case underlying bone is affected. As we know trauma is an inciting factor in osteomyelitis, patients with CIP are prone to repetitive trauma and subsequent osteomyelitis and septic arthritis. Zhang Y et al in their review of 91 Japanese patients with CIP had bone and joint infection in $24 \%$ of the patients [12]. Immunodeficiency may be another aspect that may increase the incidence of infection in these patients but not much research work has been done on this entity. There is evidence of dysfunction of adaptive immune system and defective innate immunity in CIPA patients [19]. Kilic S et al. [20] presented a report of a 7 year old girl with CIPA, recurrent infections and associated hypogammaglobulinemia.

Repeated trauma leads to recurrent and multiple fractures which are painless and hence, unnoticed. Attention of parents is drawn by the subsequent swelling or deformity. If timely diagnosis and intervention is not sought, the child continues to use the extremity and this leads to exuberant callus formation. Fractures are the most common presentation in CIP with a reported incidence of $65 \%$ and lower limbs are most commonly affected with $91 \%$ of the fractures involving the lower extremity [12]. Bar-On E et al. [21] in their series of 13 patients had a total of 31 fractures of which 28 (90.3\%) involved the lower extremity. Secondary infection may give rise to non-union of fractures and such cases are difficult to manage as surgery in CIPA patients has poor results and higher rate of complications [16]. There is also higher incidence of development of heteroptrophic ossification [22,23]. Beside fractures they are prone to dislocations. Hip joint is more commonly involved than others [24]. Zhang Y et al. [12] in their review of 91 Japanese patients had dislocations in 27 (30\%) patients with 91\% of these dislocations affecting hip joint.

Neuropathic arthopathy or charcot joint disease is a very wellknown complication seen in CIP and most commonly involves knee and ankle joints. There was an incidence of $29 \%$ with $87 \%$ of the cases involving lower extremity in the review by Zhang Y et al. [12]. As the destruction of the joint continues the joint may become unstable. Results of surgical management of neuropathic arthopathy in CIPA are poor with high rate of failure. Involvement of spine has also been mentioned in the literature, though less common than extremities and includes deformities, neuropathic arthopathy and instability which can lead to neurological complications. Decompression of the neural elements may be required and multilevel fusion is warranted in Charcot's arthopathy of the spine in case there is instability [25-27].

Cases of avascular necrosis (AVN) associated with CIP is well mentioned in the literature. AVN of femoral head, talar body, navicular and medial femoral condyle have been reported $[21,24,28]$. Deformities in CIP may be a result of physeal injury secondary to a fracture or osteomyelitis, joint destruction from septic arthritis, traumatic dislocation of joints, joint destruction and instability as a result of neuropathic arthopathy.

\section{Conclusion}

Congenital insensitivity to pain can have a varied musculoskeletal presentation. Complications and deformities are more pronounced. Results of surgical intervention are poor and plagued with complications and failures. Parent education is an important aspect in prevention and early diagnosis of these musculoskeletal problems. 


\section{References}

1. Qureshi AR, Ayaz SB, Khan AH, Gill ZA (2015) Congenital indifference to pain: a report of three cases from Pakistan. Turk J Phys Med Rehab 61: 288-292.

2. Morrison I, Perini I, Dunham J (2013) Facets and mechanisms of adaptive pain behaviour: predictive regulation and action. Front Hum Neurosci 7: 755.

3. Kurnaz R, Asci M, Balta O, Aytekin K, Gunes T (2017) Congenital insensitivity to pain syndrome accompanied by neglected orthopedic traumas and complications. Arch Clin Cases 4(1): 27-33.

4. Hirsch E, Moye D, Dimon JH (1995) Congenital indifference to pain: longterm follow-up of two cases. South Med J 88(8): 851-857.

5. Yimin Z, Xin J, Jianyong L (2014) Congenital insensitivity to pain and anhidrosis syndrome: two cases involving a brother and sister. Chin Med J 127(22): 3999-4000.

6. Dyck PJ (1993) Neuronal atrophy and degeneration predominantly affecting peripheral sensory and autonomic neurons. In: Dyck PJ, Thomas PK, Griffin JW, Law PA, Peduslo JF (Eds.), Peripheral neuropathy, ( $3^{\text {rd }}$ edn), WB Saunders Company Philadelphia, USA, pp. 1065-1093.

7. Langer J, Goebel HH, Veit S (1981) Eccrine sweat glands are not innervated in hereditary sensory neuropathy type IV. An electronmicroscopic study. Acta Neuropathol 54(3): 199-202.

8. Rosemberg S, Marie SK, Kliemann S (1994) Congenital insensitivity to pain with anhidrosis (hereditary sensory and autonomic neuropathytype IV). Pediatr Neurol 11(1): 50-56.

9. Gupta B (2003) Congenital insensitivity of pain with anhidrosis. Indian J Pediatr 70(0031): 109-111.

10. Indo Y, Tsuruta M, Hayashida Y, Karim MA, Ohta K, et al. (1996) Mutations in the TRKA/NGF receptor gene in patients with congenital insensitivity to pain with anhidrosis. Nat Genet 13(4): 485-488.

11. Montaseri Z, Rahimian M, Sobhani Q, Gheidar F, Nematollahi A, et al. (2014) Insensitivity to pain due to genetic mutation. Hormozgan Med J 18(4): 337-345.

12. Zhang Y, Haga N (2014) Skeletal complications in congenital insensitivity to pain with anhidrosis: a case series of 14 patients and review of articles published in Japanese. J Orthop Sci 19(5): 827-831.

13. Nagasako EM, Oahlander AL, Dworkin RH (2003) Congenital insensitivity to pain: an update. Pain 101(3): 213-219.

14. Kim JS, Woo YJ, Kim GM, Kim CJ, Ma JS, et al. (1999) Congenital insensitivity to pain with anhidrosis: a case report. J Korean Med Sci 14: 460-464.
15. Minde J, Svensson O, Holmberg M, Solders G, Toolanen G (2006) Orthopedic aspects of familial insensitivity to pain due to a novel growth factor beta mutation. Acta Orthop 77: 198-202.

16. Ali N, Sharma S, Sharma S, Kamal Y, Sharma S (2012) Congenital insensitivity to pain with anhidrosis (HSAN Type IV) extremely rare syndrome that can be easily missed by bone and joint surgeons: a case report. Iran J pediatr 22(4): 559-563.

17. Jun H, Ai-bin Z, Zhen L, Jiang-nan Z (2006) Congenital insensitivity to pain with anhidrosis and progressing acro-osteolysis: a case report with 7-year follow-up. Chin Med J 119:2134-2137.

18. Hatzis J, Gourgiotou K, Koumelas D (1992) Congenital sensory neuropathy with anhidrosis (hereditary sensory neuropathy type IV). Australas J Dermatol 33(2): 103-107.

19. Varma AV, McBride L, Marble M, Tilton A (2016) Congenital insensitivity to pain and anhidrosis: case report and review of findings along neuroimmune axis in the disorder. J Neurol Sci 370: 201-210.

20. Kilic SS, Ozturk R, Sarisozen B, Rotthier A, Baets J, et al. (2008) Humoral immunodeficiency in congenital insensitivity to pain with anhidrosis. Neurogenetics 10: 161-165.

21. Bar-On E, Weigl D, Parvari R, Katz K, Weitz R, et al. (2002) Congenital insensitivity to pain. Orthopaedic manifestations. J Bone Joint Surg $\mathrm{Br}$ 84(2): 252-257.

22. Abdulla M, Khaled SS, Khaled YS, Kapoor H (2014) Congenital insensitivity to pain in a child attending a paediatric fracture clinic. J Pediatr Ortho B 23(5): 406-410.

23. Erdil M, Bilsel K, Imren Y, Ceylan HH, Tuncay I (2012) Total hip arthoplasty in a patient with congenital insensitivity to pain: a case report. J Med Case Rep 6: 190.

24. Schulman H, Tsodikow V, Einhorn M, Levy Y, Shorer Z, et al. (2001) Congenital insensitivity to pain with anhidrosis (CIPA): the spectrum of radiological findings. Pediatr Radiol 31(10): 701-705.

25. Cassidy RC, Shaffer WO (2008) Charcot arthopathy because of congenital insensitivity to pain in an adult. Spine J 8(4): 691-695.

26. Sliwa JA, Rippe D, Do V (2008) Charcot spine in a person with congenital insensitivity to pain with anhidrosis: a case report of re-diagnosis. Arch Phys Med Rehabil 89(3): 568-571.

27. Tsirikos AL, Haddo O, Noordeen HH (2004) Spinal manifestations in a patient with congenital insensitivity to pain. J Spinal Disord Tech 17(4): 326-330.

28. Lopez LMP, Gozalez MC, Iglesia DG, Ricart S, Gimenez GK (2015) Update review and clinical presentation in congenital insensitivity to pain and anhidrosis. Case Rep Pediatr 2015: 589852.
Creative Commons Attribution 4.0

International License

For possible submissions Click Here

\section{Submit Article}

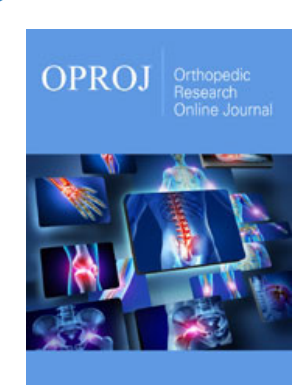

\section{Orthopedic Research Online Journal}

\section{Benefits of Publishing with us}

- High-level peer review and editorial services

- Freely accessible online immediately upon publication

- Authors retain the copyright to their work

- Licensing it under a Creative Commons license

- Visibility through different online platforms 